\title{
The combination of forskolin and VPA increases gene expression efficiency to the hypoxia/neuron-specific system
}

\author{
Zhimin Pan ${ }^{1,2,3 \#}$, Jinsoo Oh ${ }^{2,4 \#}$, Lu Huang ${ }^{5 \#}$, Zhaoxun Zeng ${ }^{3}$, Pingguo Duan ${ }^{3}$, Zhiyun Li ${ }^{3}$, Yeomin Yun ${ }^{2,4}$, \\ Janghwan $\mathrm{Kim}^{6}$, Yoon $\mathrm{Ha}^{2,4 *}$, Kai Cao ${ }^{1 *_{\wedge}}$ \\ ${ }^{1}$ Spine Center, The Second Affiliated Hospital of Nanchang University, Nanchang, China; ${ }^{2}$ Department of Neurosurgery, Spine and Spinal Cord \\ Institute, Yonsei University College of Medicine, Seoul, Republic of Korea; ${ }^{3}$ Department of Orthopedics, The First Affiliated Hospital of Nanchang \\ University, Nanchang, China; ${ }^{4}$ Brain Korea 21 PLUS Project for Medical Science, College of Medicine, Yonsei University, Seoul, Republic of Korea; \\ ${ }^{5}$ Department of Child Health and Care, Jiangxi Maternal and Child Health Hospital, Nanchang, China; ${ }^{6}$ Stem Cell Research Center, Korea Research \\ Institute of Bioscience and Biotechnology, Daejeon, Republic of Korea \\ Contributions: (I) Conception and design: K Cao, Y Ha; (II) Administrative support: J Oh, J Kim; (III) Provision of study materials or patients: Y \\ Ha, K Cao; (IV) Collection and assembly of data: Z Pan, L Huang, Z Zeng; (V) Data analysis and interpretation: Z Pan, P Duan, Z Li, Y Yun; (VI) \\ Manuscript writing: All authors; (VII) Final approval of manuscript: All authors. \\ \#These authors contributed equally to this work and are first co-authors. \\ *These authors contributed equally to this work. \\ Correspondence to: Kai Cao, MD, PhD. Spine Center, The Second Affiliated Hospital of Nanchang University, 1 Minde Road, Nanchang, China. \\ Email: kaichaw@126.com; Yoon Ha, MD, PhD. Department of Neurosurgery, Spine and Spinal Cord Institute, Yonsei University College of \\ Medicine, 50 Yonsei-ro, Seodaemun-gu, Seoul 03722, Republic of Korea. Email: hayoon@yuhs.ac.
}

Background: Spinal cord injury (SCI) tends to damage neural tissue and generate a hypoxic environment. Studies have confirmed that single therapy with gene or stem cells is inefficient, but research into combining stem cells and gene therapy in treating tissue damage has been undertaken to overcome the related limitations, which include low gene delivery efficiency and therapeutic outcome. Thus, a combination of stem cells, gene therapy, and a hypoxia-specific system may be useful for the reconstruction of SCI.

Methods: To synergistically treat SCI, a combined platform using a hypoxia/neuron-inducible gene expression system (HNIS) and human induced-neural stem cells (hiNSCs) produced by direct reprogramming was designed. Sox2- or nestin-positive hiNSCs were differentiated to Tuj1-, MAP2-, or NeuN-positive neurons.

Results: HNIS showed consistent hypoxia/neuron-specific gene expression in hiNSCs cultured under hypoxia. In particular, the HNIS-hiNSC combined platform revealed a complex pattern with higher gene expression compared with a single platform. In addition, we found that an optimal combination of small molecules, such as CHIR99021, valproic acid (VPA), glycogen synthase kinase-3 $\beta$ (GSK3 $\beta$ ), and histone deacetylase (HDAC) inhibitors, could significantly enhance gene expression with HNIS-hiNSCs in the hypoxic environment.

Conclusions: This experiment demonstrated that HNIS-hiNSCs combined with GSK3 and HDAC inhibitors may present another promising strategy in the treatment of SCI.

Keywords: Glycogen synthase kinase-3 (GSK3); histone deacetylase (HDAC); hypoxia/neuron-specific system; small molecule; spinal cord injury (SCI)

Submitted Apr 16, 2020. Accepted for publication Jun 23, 2020.

doi: $10.21037 /$ atm-20-3871

View this article at: http://dx.doi.org/10.21037/atm-20-3871

\footnotetext{
^ ORCID: 0000-0001-6384-1227.
} 


\section{Introduction}

Spinal cord injury (SCI) is a common and severe disease with a complex underlying mechanism. Therapeutic strategies for SCI include non-surgical and surgical treatments. Thereinto, the management of high-dose methylprednisolone has been controversial due to the indeterminate results (1). While intravenous administration of vasopressors and maintaining a mean arterial blood pressure after SCI could offer blood supply for the spinal cord, but does not directly recover the injured cord (2). Traumatic SCI could be treated by removing bone and disk fragments from spinal canal and realigning spine, since surgically stabilizing the spine allows for early mobilization and rehabilitation for injured spinal cord (3). However, given that it is possible for spinal cord damage to occur without fracture (3) and that predominant damage in traumatic SCI derives from cell death in the second stage (4), surgery may not be viable. Fortunately, it is not the only approach for treating SCI, and researchers have found that transplantation-based therapy can provide numerous key benefits, as grafts can be precisely delivered to relevant lesions to achieve neuroprotection (5) and support longterm cellular integration and therapeutic replacement. More specifically, researchers have demonstrated that transplantation of neural stem cells (NSCs) or neural progenitor cells shows promise in treating SCI and neurodegenerative diseases (6). In recent years, induced pluripotent stem cells (iPSCs) have been used to overcome the ethical issues of embryonic stem cells (ESCs) and to achieve autologous stem cell reprogramming from many mature cell types (7). As autologous stem cells from patients or animals, iPSCs have been intensively investigated for treating intractable diseases $(7,8)$.

Directly reprogrammed stem cells have been used for the treatment of neurodegenerative diseases, including SCI in basic research (9), and stem cell therapy has achieved effective outcomes in some preclinical studies $(10,11)$. However, the therapeutic effectiveness of stem cells for SCI still needs to be improved, as SCI has a complicated pathophysiology and possibly demands a combined strategy for adequate treatment (12-14). The therapeutic challenges include how to address a damaged nerve existing in an ischemic environment following SCI (15). Therefore, our research team developed a tissue-specific gene expression platform based on hypoxia- and neuron-specific promoters to improve ischemia in the injured spinal cord $(16,17)$. However, our previous study confirmed that a single gene delivery strategy is inefficient for SCI (16). Although cell transplantation is feasible for SCI, the efficacy remains unproven (18), and only stem cells are not significantly effective in spinal lesion treatment (19).

Both hypoxia- and neuron-specific gene expression systems (NSE) have shown that the overexpression systems can function well in the hypoxic-ischemic environment. In combination with stem cells, this special gene expression system can overcome critical hypoxic limitations, including low gene delivery efficiency. Both Azmitia et al. and our research team obtained gene overexpression and specific autologous stem cells by directly reprogramming technology with tissue-specific transcription factors or small molecule-based methods $(20,21)$. In the present study, we investigated the application of a hypoxia/neuroninducible gene expression system (HNIS) combined with human induced NSCs (hiNSCs) in a hypoxic environment. Unexpectedly, we observed that the optimal combination of HNIS-hiNSCs and small molecules could increase the gene expression efficiency of HNIS. We present the following article in accordance with the ARRIVE reporting checklist (available at http://dx.doi.org/10.21037/atm-20-3871).

\section{Methods}

\section{Cell culture and neural differentiation}

The experiments were carried out in accordance with the guidelines provided by the Yonsei University College of Medicine and Nanchang University College of Medicine. Fibroblast-derived hiNSCs were donated by the Korea Research Institute of Bioscience and Biotechnology, and hiNSCs were identified by a research article (22). Cells were maintained at $37^{\circ} \mathrm{C}$ in a humidified atmosphere containing $5 \% \mathrm{CO}_{2}$ in a culture medium consisting of neurobasal medium and advanced Dulbecco's modified Eagle's medium/nutrient mixture $\mathrm{F}-12$ at a $1: 1$ ratio, with $1 \% \mathrm{~N}-2$ supplement (100×), 2\% B-27 supplement (50×), 0.05\% bovine serum albumin, $1 \%$ penicillin/streptomycin, $1 \%$ Glutamax-I (100x), and 0.1\% 2-mercaptoethanol (1,000x; all purchased from Life Technologies, Carlsbad, CA, USA), as well as 0.1 $\mu \mathrm{M}$ A83-01 (Tocris Bioscience, Bristol, UK), $3 \mu \mathrm{M}$ CHIR99021 (Tocris Bioscience), and leukemia inhibitory factor (LIF) (1 unit; Millipore, Temecula, CA, USA), which were dissolved in dimethyl sulfoxide (SigmaAldrich, St. Louis, MO, USA). To induce the differentiation of hiNSCs, the cells were seeded on plates coated with $1 \%$ basement membrane matrix (BD Biosciences, San Jose, CA, 
USA) at a density of $1 \times 10^{4}$ cells $/ \mathrm{cm}^{2}$ and incubated at $37^{\circ} \mathrm{C}$ for $24 \mathrm{~h}$. The next day, we replaced the media containing A83-01, CHIR99021, and LIF with one containing brainderived neurotrophic factor, glial cell-derived neurotrophic factor, and neurotrophin-3 (all $10 \mathrm{ng} / \mathrm{mL}$, all purchased from Life Technologies). The medium was replaced every 2-3 days.

Mouse NSCs (mNSCs; 30-40 passages; CRL-2925, ATCC, Manassas, VA, USA) were maintained in Dulbecco's modified Eagle's medium/nutrient mixture F-12 (Gibco, Grand Island, NY, USA) supplemented with $10 \%$ fetal bovine serum (Thermo Scientific HyClone, Logan, UT, USA) and $1 \%$ penicillin streptomycin (Gibco). Cells were cultured under normoxic conditions at $37{ }^{\circ} \mathrm{C}$ in $5 \% \mathrm{CO}_{2}$. For neural differentiation of mNSCs, cells were seeded in a Matrigel (BD Biosciences, Bedford, MA, USA) coated with a 12-mm glass coverslip. The following day, cells' medium was replaced with basal media containing retinoic acid $(1 \mu M)$ and then maintained for 7 days. The medium was replaced every $2-3$ days.

\section{Small molecule treatment}

In some experiment, we investigated whether VPA affect to induce the luciferase expression of EpoNSE-iNSCs or EpoNSE-mNSC. The cells were seeded on plates coated with $1 \%$ basement membrane matrix (BD Biosciences, San Jose, CA, USA) at a density of $1 \times 10^{4}$ cells $/ \mathrm{cm}^{2}$ and incubated at $37^{\circ} \mathrm{C}$ for $24 \mathrm{~h}$. The next day, we replaced the media containing A83-01, CHIR99021, LIF and VPA. After 24 or $48 \mathrm{~h}$ in normoxia and hypoxia, we analyzed the luciferase expression level.

\section{Plasmid establishment and transfection}

The pSV-Luc, pNSE-Luc, and pEpo-SV-Luc plasmids were kindly provided by Hanyang University (Prof. Minhyung Lee). The HNIS plasmid was established in our previous study (16). Briefly, Epo cDNA was amplified using polymerase chain reaction (PCR) (Hotstart Pfu PCR PreMix, Bioneer, Daejeon, Korea) using pEpo-SV-AP (laboratory DNA stock). Two Epo fragments were used to construct the pEpo-NSE-Luc plasmid. First, the primers for PCR were 5'-MluI-Epo and 3'-NheI-AscI-Epo. The amplified Epo enhancer was inserted into the MluI and NheI sites upstream of the NSE promoter. The second PCR of the Epo enhancer was conducted using the primers 5'-KpnI-SpeI-EcoRV-Epo and 3'-SacI-PmeI-Epo. The
PCR products of Epo were inserted into KpnI and SacI.

For transfection, hiNSCs (30-40 passages) were seeded at a density of $1 \times 10^{5}$ cells $/ \mathrm{cm}^{2}$ in a multiwell plate and cultured under normoxic conditions $\left(21 \% \mathrm{O}_{2}\right)$. Plasmid (250 ng plasmid $\left./ \mathrm{cm}^{2}\right)$ and ViaFect transfection reagent $(3 \mu \mathrm{L}$ ViaFect/1 $\mu$ g plasmid, Promega, Madison, WI, USA) were added to $50 \mu \mathrm{L}$ of serum-free media. The ViaFect/plasmid complexes were briefly mixed and incubated at room temperature for $10 \mathrm{~min}$. The ViafFect/plasmid mixture was then added to cells and maintained under normoxic conditions for $24 \mathrm{~h}$. The next day, we changed the culture media to one containing small molecules [A83-01, $0.1 \mu \mathrm{M}$; CHIR99021, 3 HM; LIF, 1 unit; valproic acid (VPA), 1 mM] and then transferred the cells to hypoxic conditions $\left(1 \% \mathrm{O}_{2}\right)$. After $24 \mathrm{~h}$, the luciferase expression level was analyzed.

\section{Luciferase expression detector}

A luciferase expression detector (LED; Xenogen, Alameda, CA, USA) was used to measure the expression level of luciferase in terms of promoter activity in HNIS. For the in vitro study, D-luciferin $(150 \mu \mathrm{g} / \mathrm{mL})$ was added into each well and then kept in the dark for $10 \mathrm{~min}$. The cell culture plates were transferred to the LED imaging chamber, and the bioluminescence was then measured for 10-30 s.

For the in vivo study, D-luciferin $(30 \mathrm{mg} / \mathrm{kg})$ was intraperitoneally injected before anesthesia. After $10 \mathrm{~min}$, both ketamine $(100 \mathrm{mg} / \mathrm{kg}$, Yuhan, Seoul, Korea) and Rompun (10 mg/kg, Bayer Korea, Seoul, Korea) were injected to induce anesthesia (the above anesthetic drugs were used at the suggestion of our institution). The spinal cord tissues containing transplanted human induced NSCs (hiNSCs) were harvested from the animals. After washing with saline, the tissues were transferred to culture media containing D-luciferin $(300 \mu \mathrm{g} / \mathrm{mL})$ and then kept in the dark for $10 \mathrm{~min}$. The samples were transferred to the LED chamber, and the bioluminescence was then measured for 10-30 s. The luciferase level was quantified using the LED software (Xenogen, Alameda, CA, USA). For blinding analysis, different researchers performed the analysis for the LED imaging study.

\section{Immunofluorescence staining}

Samples were washed with phosphate-buffered saline (PBS, HyClone), and the cells were then fixed using 4\% paraformaldehyde ( $\mathrm{pH}$ 7.2, Millipore, Bedford, MA, USA) for $10 \mathrm{~min}$ at $20^{\circ} \mathrm{C}$. The samples were washed 3 times for 
3 min with $0.3 \%$ Tween 20 (Life Technologies) in phosphate-buffered saline with Tween20 (PBST) and incubated in blocking buffer for $30 \mathrm{~min}$ at $20^{\circ} \mathrm{C}$. Primary antibodies against Nestin (1:1,000, Millipore), Sox-2 (1:250; Abcam, Cambridge, MA, USA), rabbit anti-Tuj1 (1:1,000, Abcam), NeuN (1:1,000, Millipore), MAP2 (1:1,000, Millipore), Synapsin-1 (1:205, Abcam), and neurofilament (1:1,000, Abcam) were diluted in blocking buffer and allowed to react with the samples for $60 \mathrm{~min}$ at $20^{\circ} \mathrm{C}$.

The samples were washed with PBST 3 times ( $3 \mathrm{~min}$ each). Cy3- or FITC-conjugated secondary antibodies (1:500) were also diluted in blocking buffer and incubated in the dark for $30 \mathrm{~min}$ at $20^{\circ} \mathrm{C}$. After 3 washes with PBST, the samples were stained using 4',6'-diamino-2-phenylindole (Vector Laboratories, Burlingame, CA, USA).

\section{Flow cytometry}

After removing the medium and washing with PBST, the cells were detached from the culture plates using $0.25 \%$ trypsin/ethylenediaminetetraacetic acid (EDTA) (Life Technologies) and harvested in 1.5-mL Eppendorf tubes (Eppendorf, Hamburg, Germany). Fixation was performed with $4 \%$ paraformaldehyde for $10 \mathrm{~min}$, and the samples were then washed twice with PBST for $3 \mathrm{~min}$. Then, the cells were incubated with primary antibodies against Nestin $(1: 1,000)$ and Sox-2 $(1: 250)$ for $60 \mathrm{~min}$ at $20^{\circ} \mathrm{C}$. Following 3 washes with PBST, the cells were incubated with secondary antibodies in the dark for $30 \mathrm{~min}$ at $20^{\circ} \mathrm{C}$. Finally, the pellet was washed in $200 \mu \mathrm{L}$ PBST 3 times. Samples were analyzed using a FACSCalibur flow cytometer (Becton Dickinson, Rutherford, NJ, USA) and Cell Quest software (Becton Dickinson).

\section{SCI and cell transplantation}

For transplantation of iNSCs transfected with the HNISLuc plasmid, the cells (at a density of $1 \times 10^{5} \mathrm{cells} / \mathrm{cm}^{2}$ ) were seeded in Matrigel-coated $60-\mathrm{mm}$ dishes and then cultured under normoxic conditions $\left(21 \% \mathrm{O}_{2}\right)$. The HNIS plasmids (250 $\mathrm{ng}$ plasmid $/ \mathrm{cm}^{2}$ ) were transfected into hiNSCs using the previously mentioned transfection protocol and then maintained under normoxic conditions for $24 \mathrm{~h}$. On the next day, we changed the culture media to one containing small molecules (A83-01, 0.1 $\mu \mathrm{M}$; CHIR99021, $3 \mu \mathrm{M}$; LIF, 1 unit; VPA, $1 \mathrm{mM}$ ) and then transferred the cells to hypoxic conditions $\left(1 \% \mathrm{O}_{2}\right)$. After $24 \mathrm{~h}$, cells were harvested using trypsin and then resuspended in PBST at a density of

\section{$2.5 \times 10^{5}$ cells $/ \mu \mathrm{L}$.}

For inducing anesthesia, both ketamine $(100 \mathrm{mg} / \mathrm{kg}$, Yuhan) and Rompun (10 mg/kg, Bayer Korea) were intraperitoneally injected into C57BL/6 mice (male, 6-week-old, 30 g; OrientBio, Seongnam, Gyeonggi-do, Korea). We performed a laminectomy at thoracic level 11; SCI was induced using a self-closing forcep (compression injury for $1 \mathrm{~s}$ ).

We divided above mice into 2 groups using a simple randomization method: Group 1, HNIS-NSCs $(n=10)$; Group 2, HNIS-iNSCs with small molecules $(n=10)$. Subsequently, the transfected cells $\left(5 \times 10^{5}\right.$ cells, $\left.2 \mu \mathrm{L}\right)$ were immediately injected into the SCI site at a $1 \mu \mathrm{L} / \mathrm{min}$ injection rate using a syringe pump (KDS 310 Plus, KD Scientific, Inc., Holliston, MA, USA). Cyclosporin A (10 mg/kg, Chong Kun Dang, Seoul, Korea) and cefazolin (20 mg/kg, Yuhan) were also intraperitoneally injected. Kerorolac $(0.1 \mathrm{mg} / \mathrm{kg})$ was intraperitoneally injected to minimize animal suffering.

Our protocols were approved by the Animal Care and Use Committee of the Medical Research Institute at Yonsei University Health System. The experimenter was unaware of the animal's group during experimentation and statistical analysis. This randomized animal experiment was conducted in accordance with international guidelines on the ethics of animals, and we made efforts to minimize the number of animals used.

\section{Statistical analysis}

Statistical analysis was performed with SAS version 9.4 and SPSS version 22 (IBM; Chicago, IL, USA). Student's $t$-test was performed to assess the differences between the two groups. One-way analysis of variance and Tukey's post hoc test were performed to assess differences among the two groups. Normally distributed data are presented as the mean (standard deviation), and a $\mathrm{P}$ value less than 0.05 was considered statistically significant.

\section{Results}

\section{biNSC characterization}

We first performed characterization, including the assessment of proliferation and marker expression of the hiNSCs produced by direct reprogramming. The hiNSCs showed a rosette morphology and rapid growth (Figure S1), and most of the cells were positive for Ki67 in complete 
growth medium (Figure 1A,B,C). At this stage, $>90 \%$ of the hiNSCs were positive for Nestin and Sox-2 (Figure 1D,E).

Next, we investigated the neural differentiation potency of the hiNSCs in vitro and in vivo. In the neural differentiation medium, many of the hiNSCs differentiated into neurons, which were positive for Tuj1, MAP2, and NeuN 2 weeks after induction of neural differentiation (Figure 1F). Furthermore, green fluorescent protein (GFP)expressing hiNSCs were transplanted into the injured spinal cord and maintained from 1 week to 8 weeks. At 1 week, we confirmed that many of the hiNSCs had survived in the injured spinal cord tissue, and these were positive for human-specific nuclei and Nestin (Figure 1G). At 8 weeks, some of the hiNSCs remained Nestin-positive (Figure 1H), and many of the hiNSCs differentiated into MAP2-, Synapsin-1- and NeuN-positive cells (Figure 1I). These results indicate that the hiNSCs used in these experiments are indeed NSCs.

\section{Gene expression pattern of HNIS in biNSCs}

Next, we examined the function of HNIS in hiNSCs maintained under normoxia or hypoxia. To investigate neuron-specific expression, we assessed luciferase expression in hiNSCs transfected with SV- or NSE-systems. In LED imaging, the hiNSCs transfected with NSE showed high gene expression compared with the Simian virus (SV) system (Figure 2A). This implies that the NSE system is more specific to NSCs than the SV system. Furthermore, we designed the HNIS by combining the Epo enhancer and NSE promoter. To confirm the hypoxia/neuronspecific expression, we compared the luciferase expression level in the hiNSCs transfected with the NSE or HNISs under normoxia or hypoxia. In LED imaging, the hiNSCs transfected with HNIS showed a higher gene expression level than that of the NSE system under hypoxia (Figure 2B). Next, we verified the gene expression level by switching the oxygen concentration (i.e., from normoxia to hypoxia and from hypoxia to normoxia). We again confirmed that gene expression controls depended on oxygen concentration (Figure 2C,D,E).

We applied the HNIS to mNSCs to verify hypoxia/ neuron-specific gene expression in different types of NSCs. The mNSCs were also positive for Sox-2 (Figure $2 F$ ) and could be differentiated into Tuj1-, MAP2-, and neurofilament-positive cells in neural differentiation medium containing retinoic acid (Figure 2G). Therefore, we demonstrated that the HNIS functions optimally in different types of NSCs (Figure 2H,I).

\section{Treatment with a bistone deacetylase (HDAC) inbibitor improves the efficiency of HNIS}

Figure 2 shows the results confirming the specificity of HNIS to both hypoxic environments and neuronal cells. Next, we performed an experiment to identify small molecules that may improve the efficiency of HNIS. First, we assessed whether VPA treatment increases the gene expression level in hiNSCs transfected with the NSE system (NSE-iNSCs). To this end, VPA, a HDAC inhibitor, was added to the complete iNSC culture medium containing A83-01, LIF, and CHIR99021. The luciferase expression level was significantly increased in the VPA-treated group (Figure $3 A$ ), and this phenomenon was also observed with the HNIS group (Figure $3 B, C$ ). We also compared the gene expression level between HNIS and NSE under hypoxia; the HNIS showed an increased gene expression pattern compared with the NSE system (Figure 3D). Finally, we verified the combination effect of HNIS and small molecules in a SCI model. The HNIS-hiNSCs were transplanted into the injured spinal cord after treatment with VPA for $24 \mathrm{~h}$ under hypoxia. Luciferase expression was increased in the VPA-treated group (animal experiment, Figure 3E). These results thus imply that the combination of VPA with A83, LIF, and CHIR99021 is critical to increasing the gene expression in HNIS.

\section{The combination of GSK3 and HDAC inbibitors increases luciferase expression in HNIS}

Figure 3 illustrates the results confirming that VPA treatment with complete media containing A83-01, LIF, and, CHIR99021 increases the gene expression level in HNIS-hiNSCs. However, it is unclear which molecule combination plays a role in increasing gene expression in HNIS. Therefore, we further screened small molecule combinations that may be pivotal in increasing the gene expression in HNIS. First, we excluded single small molecules, one by one, in mixed combinations. The luciferase expression level was remarkably decreased in the absence of CHIR99021 or VPA (Figure 4A, B,C,D). This phenomenon was observed in other hiNSCs derived from different donors and in the combination of VPA and LiCl. These results indicate that CHIR99021 or VPA play a pivotal role in controlling gene expression and that the combination of CHIR99021 and VPA is synergistic. 


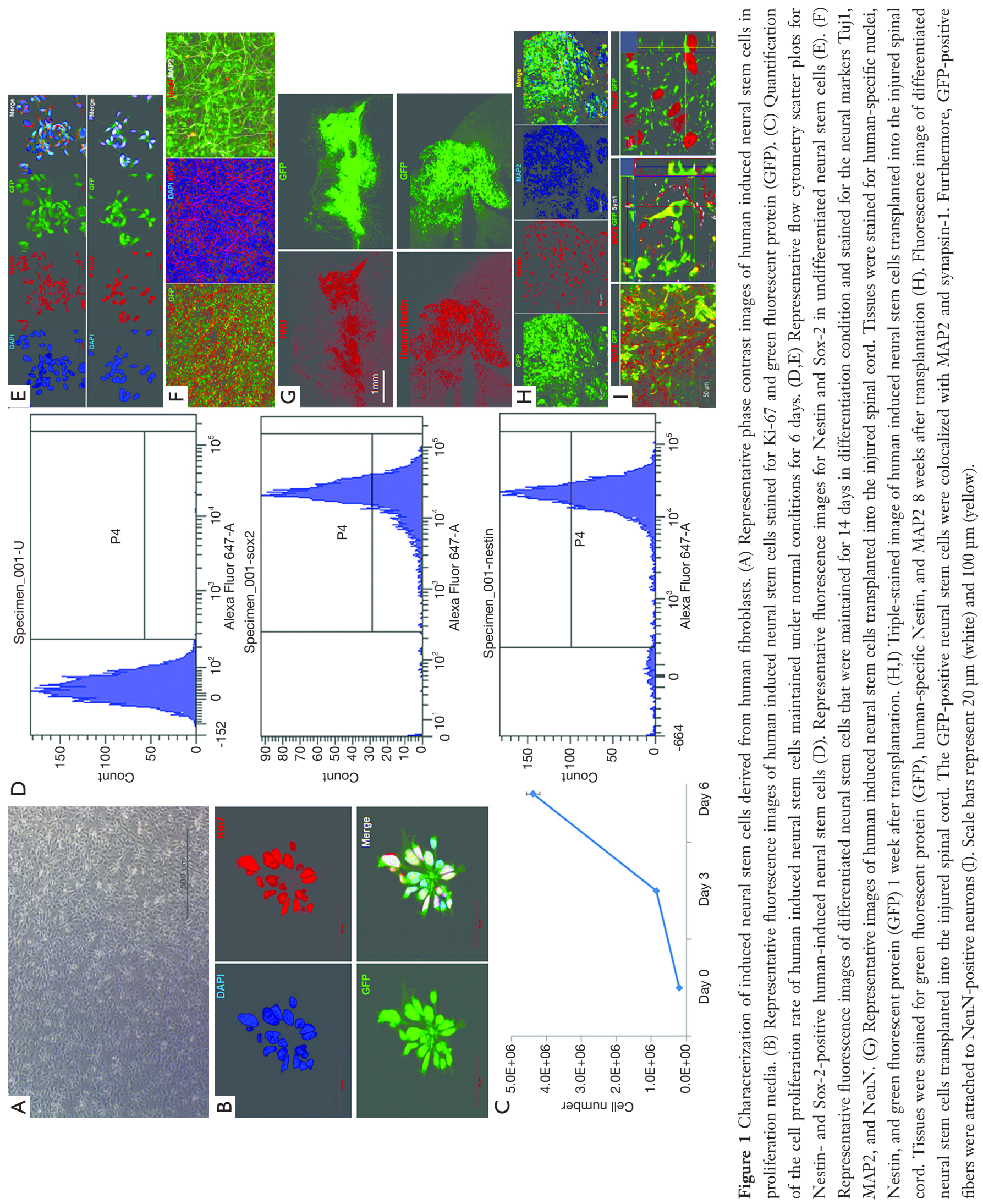



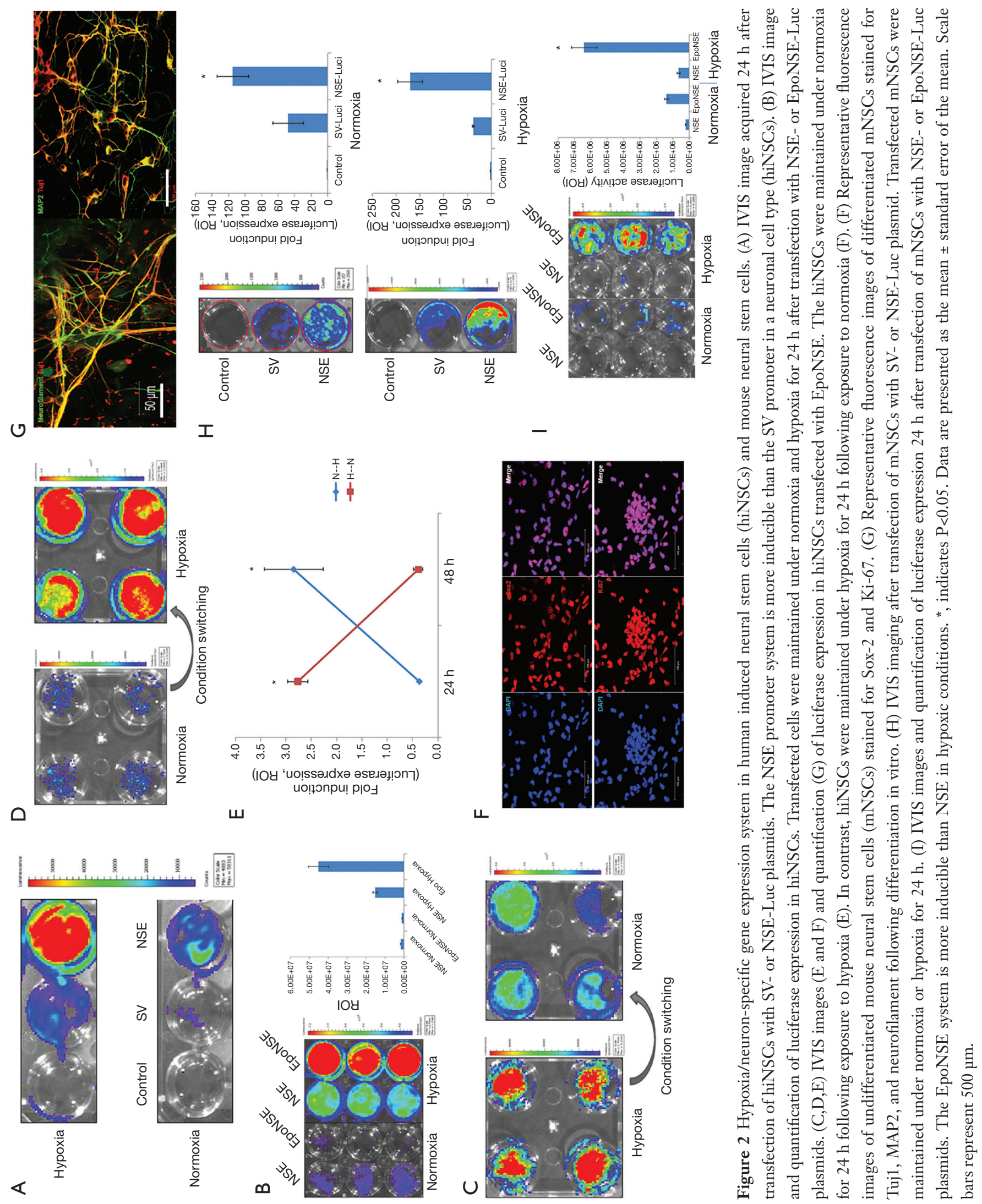


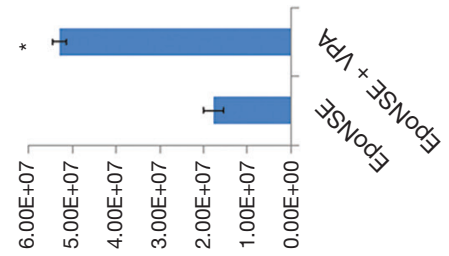

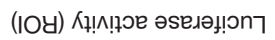

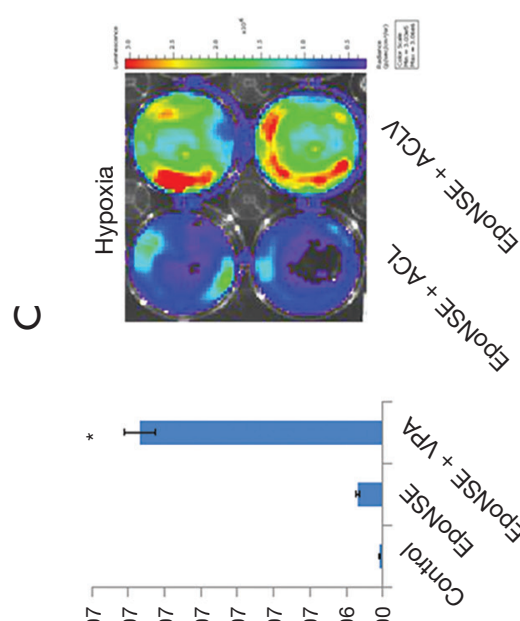

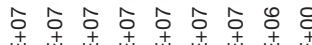

岁岩岩岩
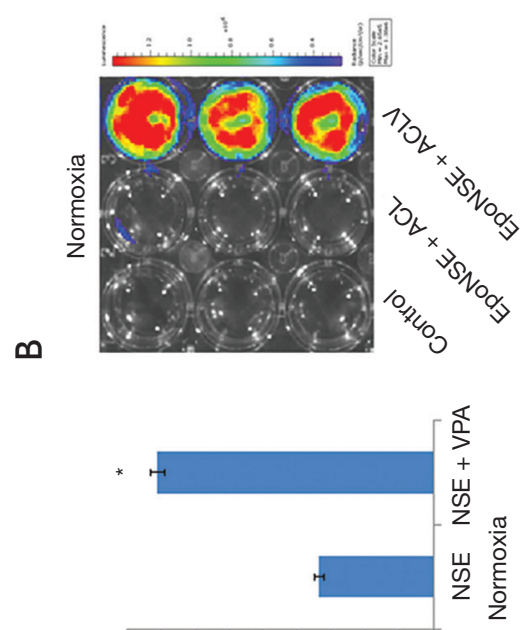

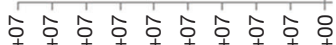

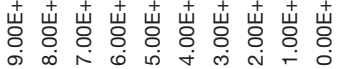

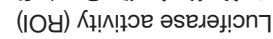

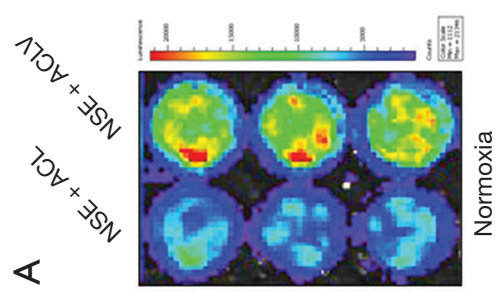

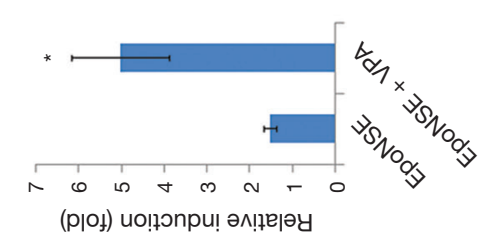
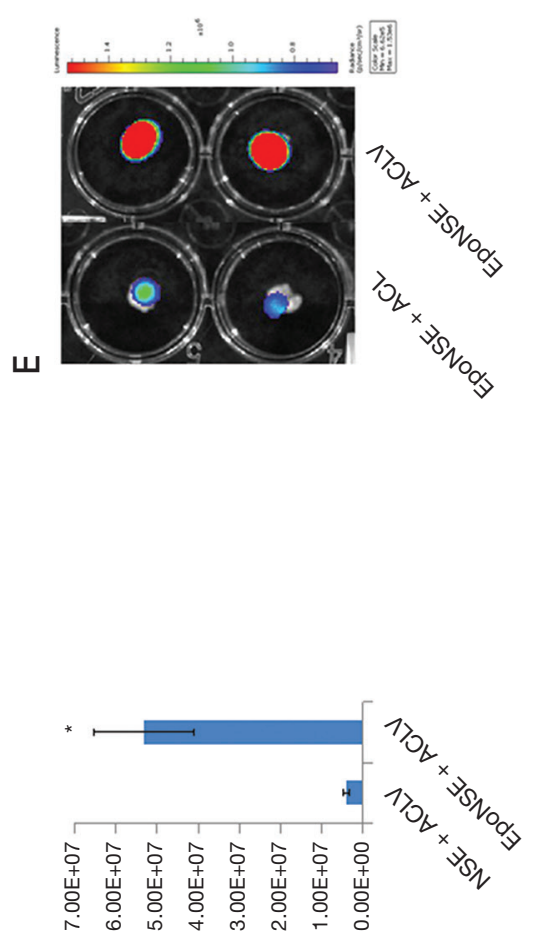

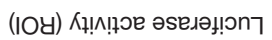

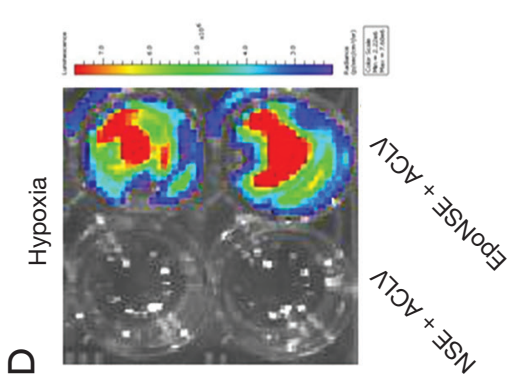

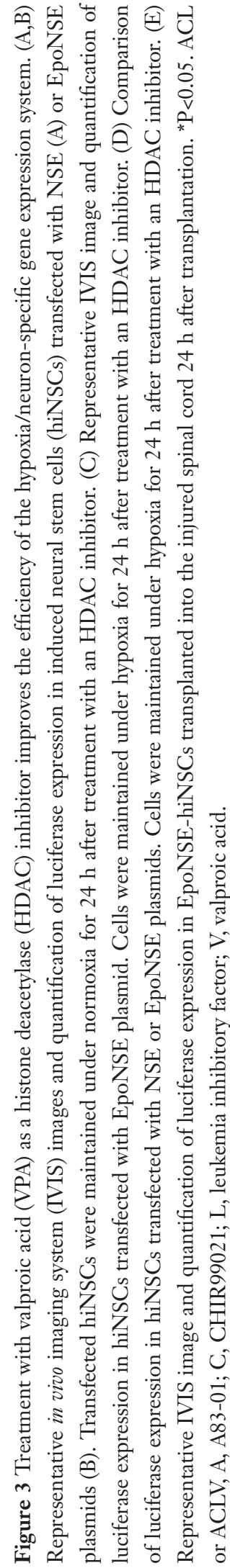

(c) Annals of Translational Medicine. All rights reserved. 

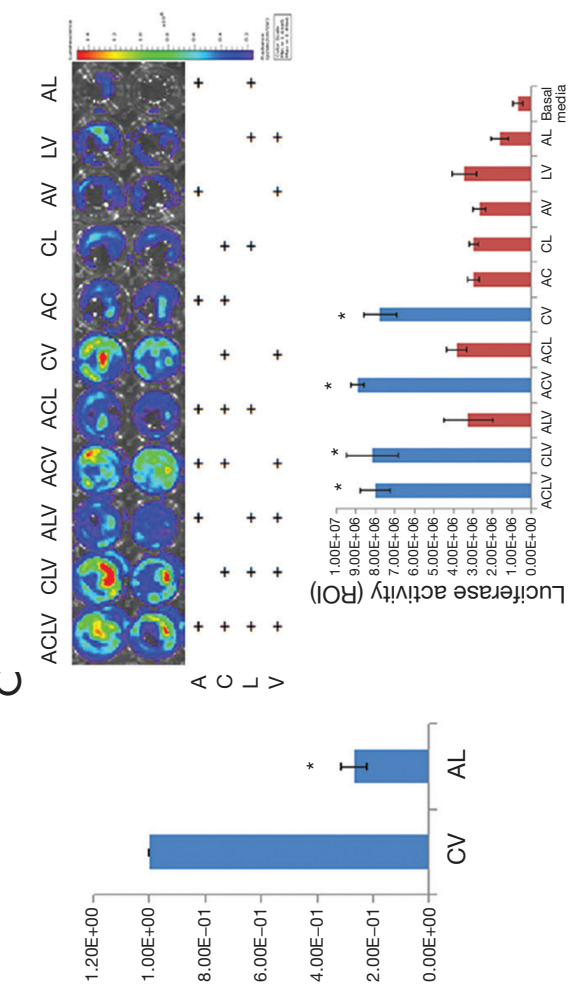

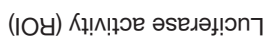

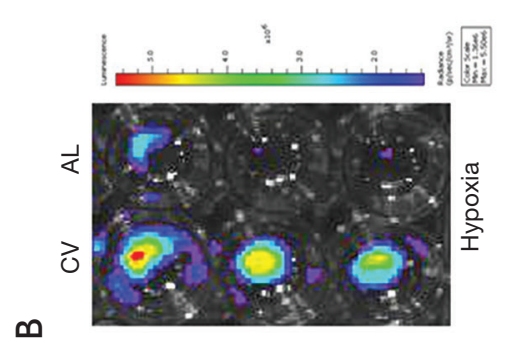

$\infty$
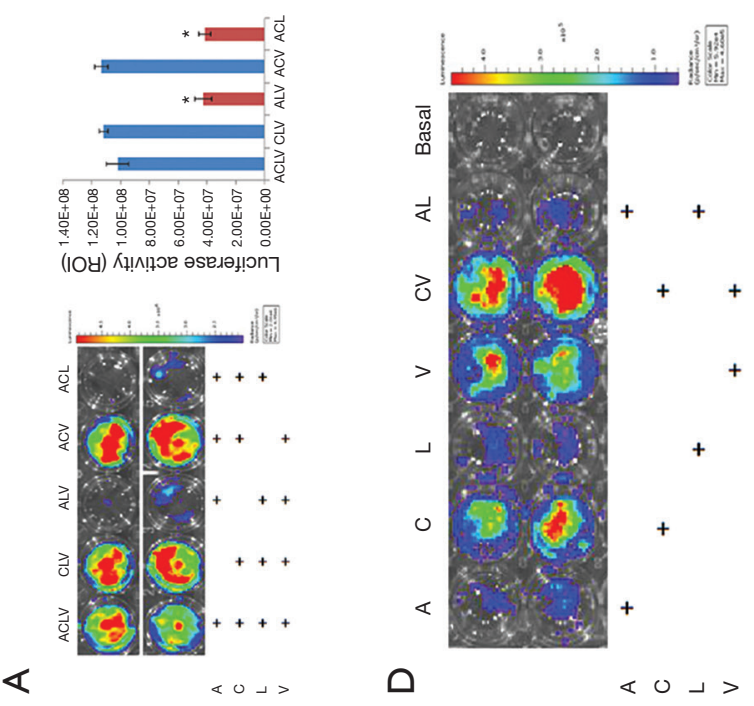

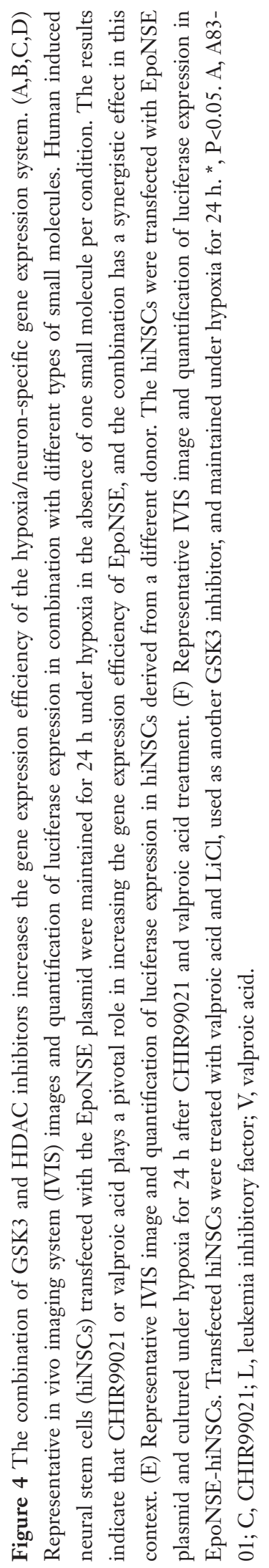



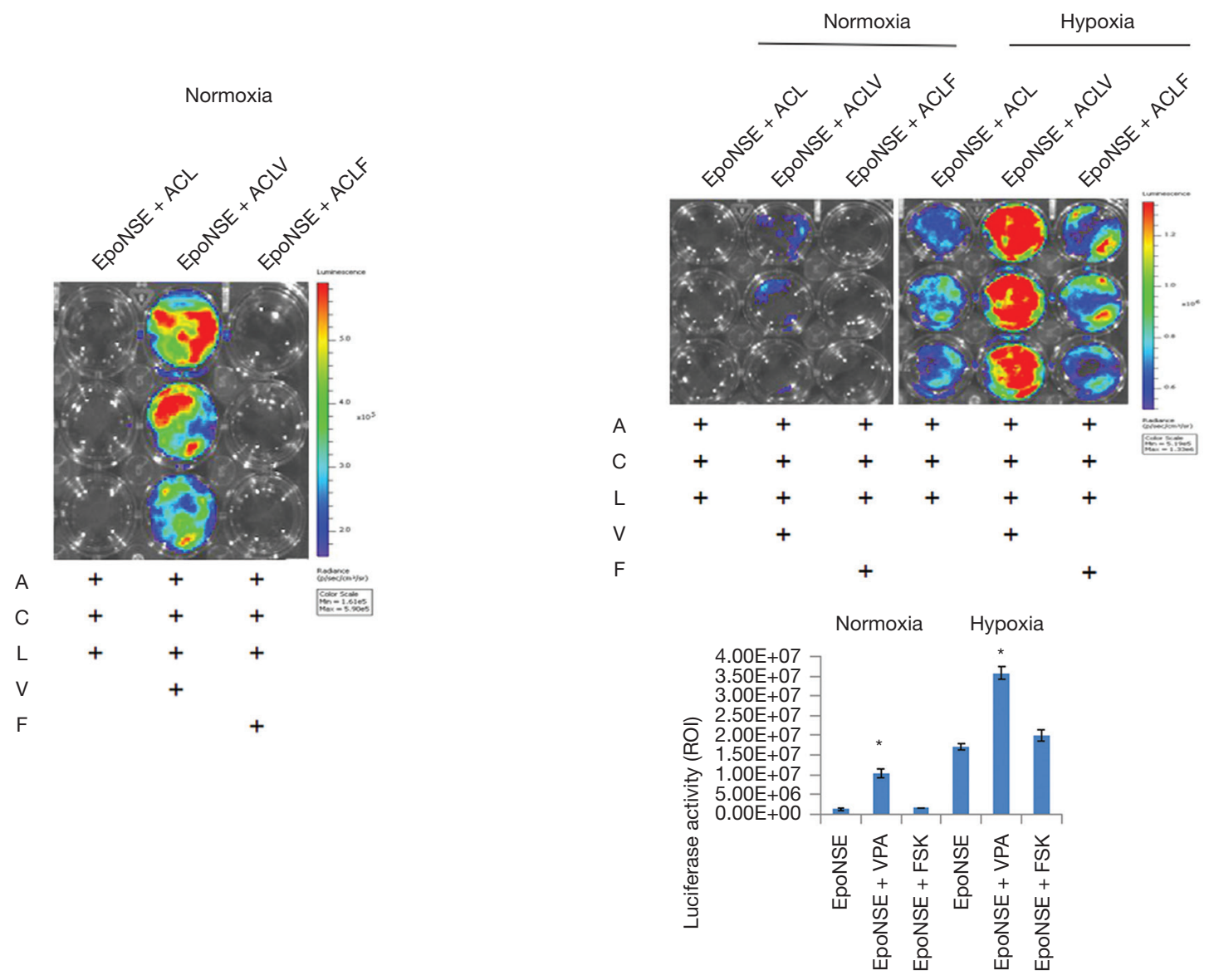

Figure 5 An HDAC inhibitor rapidly increases the activity of EpoNSE in combination with a GSK3 inhibitor rather than forskolin as a common neural differentiation inducer. Representative IVIS image of luciferase expression in EpoNSE-hiNSCs treated with different types of small molecules in combination with valproic acid and forskolin. This result indicates that combination with forskolin, a neural differentiation inducer, does not show a synergistic effect within a short time, unlike valproic acid. *, P<0.05. A, A83-01; C, CHIR99021; L, leukemia inhibitory factor; $\mathrm{V}$, valproic acid; F, forskolin.

Forskolin is widely used for the neural differentiation of NSCs. Thus, we investigated whether forskolin treatment increases the gene expression level in hiNSCs transfected with HNIS (HNIS-iNSCs). We added forskolin or VPA to complete iNSC culture media containing A83-01, LIF, and CHIR99021 and then compared the gene expression level. In LED imaging, the luciferase expression level was significantly increased in the VPA-treated group. In contrast, this increasing phenomenon was not observed for the combination of forskolin with A83-01, LIF, and CHIR99021 (Figure 5). These results indicate that the combination of forskolin, a common neural differentiation inducer, has no synergistic effect on increasing the gene expression efficiency.
To investigate whether a combination of CHIR99021 and VPA shows specific activity in the NSE system only, we compared the gene expression level in the SV or NSE systems after treatment with CHIR99021 and VPA. Treatment with VPA increased luciferase expression in both the NSE and SV systems (Figure 6A), and this phenomenon was also observed in hiNSCs derived from different donors (Figure 6B). Thus, these results indicate that the combination effect of CHIR99021 and VPA is not specific to the NSE promoter.

\section{Discussion}

A clinical study analyzing 63,109 patients with acute 

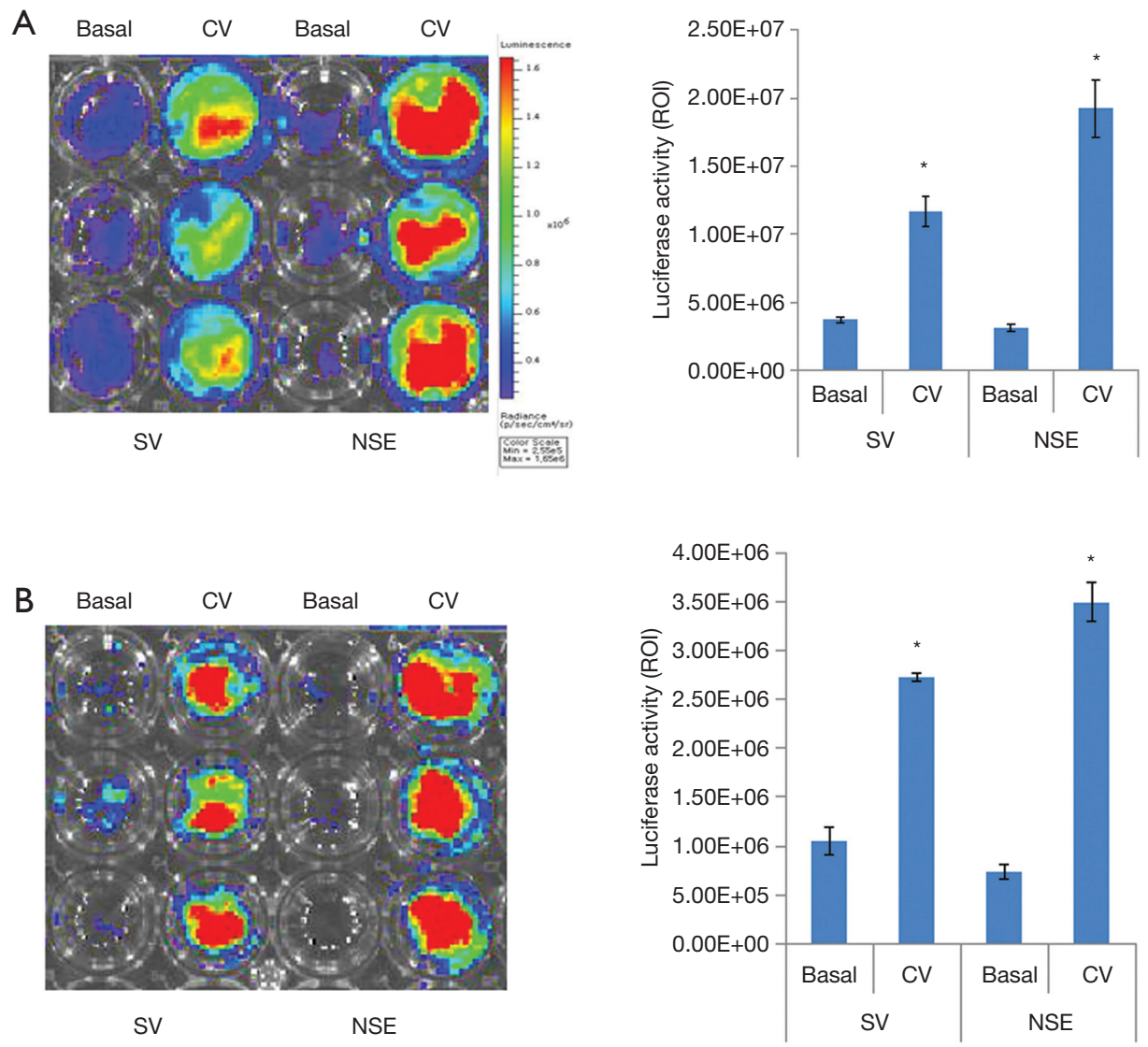

Figure 6 The combination of GSK3 and an HDAC inhibitor also increases the activity of common promoter as well as a NSE promoter. $(\mathrm{A}, \mathrm{B})$ Representative IVIS images and quantification of luciferase expression in induced neural stem cells (hiNSCs) transfected with SV or NSE plasmids. This experiment was performed in two different types of iNSCs; hiNSCs were maintained under hypoxia for 24 h after CHIR99021- and valproic acid-treatment. This result indicates that the combination of two small molecules is suitable for a common promoter and is not specific to the NSE promoter. *, $\mathrm{P}<0.05$. C, CHIR99021; V, valproic acid.

traumatic SCI from 1993 to 2012 revealed that the SCI population is increasing in the USA (23). SCI treatment is difficult because the condition is closely associated with an extended period caused by post-traumatic cell death and neurological deficits (24), and secondary degeneration after SCI requires an even longer time for adequate management (6). To maximally restrict the extent of secondary injury following SCI, transplantation of stem cells and pharmacological treatment are considered valuable approaches for preventing continuous cell injury and neural functional loss $(25,26)$.

Currently, direct reprogramming technology is being actively studied in the field of regenerative medicinal research $(27,28)$, and the direct conversion of skin fibroblasts to neurons through a combination of small molecules is particularly appealing (29). In our previous study, we observed that the efficiency of the NSE system was increased in mouse neural stem cells (mNSCs) (30). However, the hypoxic-ischemic environment within the spinal cord after SCI is detrimental to therapeutic effectiveness, while the amount of preserved spinal cord tissue is closely related to post-traumatic neural functional recovery, and even small improvements in neural protection against secondary destruction could significantly enhance functional recovery (31). In the present study, therefore, we employed a hypoxia/neuron-specific gene expression system (HNIS) that was specific for the SCI environment with a hypoxic status. Additionally, the gene expression efficiency of HNIS was significantly improved in combination with small molecules, including glycogen synthase kinase- $3 \beta$ 
(GSK3 $\beta$ ) and HDAC inhibitors.

\section{Gene and stem cells synergistically increase gene delivery efficiency}

Given that SCI leads to an ischemic environment with nerve damage in the spinal cord, a hypoxia-inducible system has the potential to be applied in SCI treatment. However, our previous study confirmed that the single gene delivery strategy is inefficient except when after employing stem cells as the gene delivery system in vivo (16). Therefore, a combination strategy is the superior choice to overcome the limitations of gene delivery alone. In our previous study, a NSE system was used to enhance the gene expression efficiency in a neural lineage, such as mouse adult NSCs (17), since the first intron of NSE gene, as a strong enhancer, can confer a high level of neuron-specific expression in neuronal cells (32). In another previous study, we also identified that exogenous NSE promoter actively increased gene expression not only in terminally differentiated neurons but also in NSCs (16). However, hiNSCs have more advantages, such as their autologous nature, compared to adult NSCs. In addition, hiNSCs exhibited a high reproducibility in combination with HNIS, and the experiment proved that pEpo-NSE consistently shows hypoxia/neuron-specific gene expression in NSCs (16). As a glycoprotein hormone and an enhancer, erythropoietin (Epo) was produced in response to a low blood oxygen concentration (33), and it has been used to increase gene expression within hypoxia (34). In fact, Epo was the starting point for research of the oxygen sensing pathway (33).

\section{Small molecules, including HDAC and GSK3 inbibitors, used in stem cell research}

Recently, small molecules have been widely used in stem cells or reprogramming research fields. Small molecules have been verified to facilitate cell reprogramming and regulate cell fate, and they have cell-permeable and nonimmunogenic attributes (35). Abematsu et al. employed the restorative benefits of exogenous NSCs and VPAinduced neuronal differentiation to treat SCI (36). Their study demonstrated that grafted NSCs predominantly differentiated into neurons after daily VPA injection for 7 days. Other research teams have found that the combination of small molecules with inhibitors of transforming growth factor beta (TGF), HDAC, and GSK3 signaling could efficiently convert skin fibroblasts to
NSCs $(37,38)$. In addition, a combination of A83-01 and CHIR99021 has been used to generate neural progenitor cells from iPSCs (39). In particular, the combination of CHIR99021 and VPA could affect neural reprogramming and the regulation of promoter activity (40).

Among the small molecules used in the present study, A83-01 is a TGF- $\beta$ kinase/activin receptor-like kinase inhibitor, and LIF is a factor used for maintaining ESC pluripotency by acting downstream of the phospho-ERK pathway to block ESC differentiation. This molecule was found to restore cell viability in combination with a GSK3 inhibitor, which could improve ESC propagation and maintain pluripotency by activating $\mathrm{Wnt} / \beta$-catenin signaling $(38,41)$. Yu et al. revealed that a combination of A83-01 and LIF is effective in maintaining and generating iPSCs (42). As a multifunctional inhibitor of both HDAC and GSK3, VPA induces neuronal differentiation and promotes neuroprotection and neurogenesis by activating the ERK pathway and inducing the neurotrophic effects mediated by this pathway $(24,43)$. This beneficial effect on neurogenesis has encouraged researchers to explore the combination effect of VPA and stem cell therapy in an effort to promote neuronal differentiation of stem cells grafted in injured spinal cords $(36,44)$. Furthermore, VPA has been demonstrated to suppress ischemia-induced neuronal caspase- 3 activation (45). In addition to preventing the permeability of the blood-spinal cord barrier, VPA can attenuate the extensive inflammation, neurotoxicity, and autophagy observed during secondary injury in SCI (46). By preventing pathological autophagy from exacerbating secondary cell death, VPA exerts potent neuroprotective benefits following SCI (47). Additionally, Li et al. demonstrated that another GSK3 inhibitor, CHIR99021, can convert human ESCs to neuroepithelium in vitro (48).

In the current study, some similarities between the inhibition of HDAC and GSK3 were observed: both HDAC and GSK3 $\beta$ inhibition can upregulate the expression of neuroprotective and neurotrophic factors in the context of SCI $(49,50)$. The underlying mechanism may be associated with multiple interconnected signaling pathways mediated by the potent effects of VPA on HDAC and GSK3 inhibition. Jin et al. demonstrated that combined treatment with VPA and hypothermia led to enhancement of neuroprotective effects since hypoxia-inducible factor$1 \alpha$ and phospho-GSK3 $\beta$ expression are synergistically affected (the number of viable cells increased by 17.6\%) (51). In addition, Huang et al. revealed that inhibition of GSK$3 \beta$ and HDAC by urocortin resulted in decreased caspase- 3 
activation, and treatment with a caspase inhibitor deterred dopaminergic neuronal degeneration (52). Leng et al. further found that the synergistic neuroprotective effects of HDAC and GSK3 inhibition might be warranted for clinical trials against glutamate-related neurodegenerative diseases such as stroke, Alzheimer's disease, Parkinson's disease, and SCI (53). Apart from enhancing the expression level of target genes, combinations with TGF- $\beta$, GSK3, and HDAC inhibitors have been successfully used in studies to investigate cell reprogramming $(54,55)$.

In fact, we applied forskolin in this study because it was widely used in studies on neural differentiation or direct reprogramming. In a previous study, we confirmed that glioma cells can be converted to neurons by treatment with CHIR99021 and forskolin for 5 weeks (20). In our preliminary study, hiNSCs were frequently differentiated into neurons within 7 days of being treated with forskolin. Thus, we hypothesized that a combination of CHIR99021 and forskolin can improve the gene expression efficacy of the HNIS in hiNSCs by inducing neuronal differentiation. However, the combination with forskolin did not enhance the gene expression level of HNIS within 24 hours. This result indicates that the combination of VPA and CHIR99021 rapidly enhances promoter activity and has a synergistic effect on increasing the gene expression efficiency in HNIS.

\section{Conclusions}

This experiment demonstrated that HNIS has consistent hypoxia/neuron-specific gene expression in hiNSCs cultured under hypoxia. A complex pattern of HNIShiNSCs possesses higher gene expression compared with a single platform, such as the NSE. In addition, an optimal combination of small molecules, including GSK3 and HDAC inhibitors, combined with HNIS-hiNSCs may present a promising strategy in the treatment of SCI.

\section{Acknowledgments}

We thank the reviewers and editors for their helpful comments on this article.

Funding: The present study was supported by the National Nature Science Fund of China (No. 81860473), the 5511 Innovation-driven Program of Jiangxi Province Department of Science and Technology (No. 2165BCB18017), the Program of Health Commission of Jiangxi Province (No. 20191029), the Basic Science Research Program through the NRF, China Scholarship Council (No. 2017-3109/201708260068), and the Korean Ministry of Education (No. 2018R1D1A1B07044055).

\section{Footnote}

Reporting Checklist: The authors have completed the ARRIVE reporting checklist. Available at http://dx.doi. org/10.21037/atm-20-3871

Data Sharing Statement: Available at http://dx.doi. org/10.21037/atm-20-3871

Conflicts of Interest: All authors have completed the ICMJE uniform disclosure form (available at http://dx.doi. org/10.21037/atm-20-3871). ZP reports grants, personal fees and other from China Scholarship Council, during the conduct of the study; JO and YH report grants from NRF, grants from Korean Ministry of Education, during the conduct of the study; KC reports grants from the National Nature Science Fund of China, grants from Jiangxi Province Department of Science and Technology, grants from Health Commission of Jiangxi Province, during the conduct of the study. The other authors have no conflicts of interest to declare.

Ethical Statement: The authors are accountable for all aspects of the work in ensuring that questions related to the accuracy or integrity of any part of the work are appropriately investigated and resolved. The study was approved by the Institutional Review Board of the Second Affiliated Hospital of Nanchang University. The protocols were approved by the Animal Care and Use Committee of the Medical Research Institute at Yonsei University Health System. The experimenter was unaware of the animal's group during experimentation and statistical analysis. This randomized animal experiment was conducted in accordance with international guidelines on the ethics of animals, and the authors made efforts to minimize the number of animals used.

Open Access Statement: This is an Open Access article distributed in accordance with the Creative Commons Attribution-NonCommercial-NoDerivs 4.0 International License (CC BY-NC-ND 4.0), which permits the noncommercial replication and distribution of the article with the strict proviso that no changes or edits are made and the original work is properly cited (including links to both the 
formal publication through the relevant DOI and the license). See: https://creativecommons.org/licenses/by-nc-nd/4.0/.

\section{References}

1. Bracken MB, Shepard MJ, Holford TR, et al. Administration of methylprednisolone for 24 or 48 hours or tirilazad mesylate for 48 hours in the treatment of acute spinal cord injury: results of the Third National Acute Spinal Cord Injury Randomized Controlled Trial: National Acute Spinal Cord Injury Study. JAMA 1997;277:1597-604.

2. Ryken TC, Hurlbert RJ, Hadley MN, et al. The acute cardiopulmonary management of patients with cervical spinal cord injuries. Neurosurgery 2013;72:Suppl 2:84-92.

3. Ropper AE, Ropper AH. Acute spinal cord compression. N Engl J Med 2017;376:1358-69.

4. McDonald JW, Becker D. Spinal cord injury: promising interventions and realistic goals. Am J Phys Med Rehabil 2003;82:S38-49.

5. Lepore AC, Rauck B, Dejea C, et al. Focal transplantationbased astrocyte replacement is neuroprotective in a model of motor neuron disease. Nat Neurosci 2008;11:1294-301.

6. Li K, Javed E, Scura D, et al. Human iPS cell-derived astrocyte transplants preserve respiratory function after spinal cord injury. Exp Neurol 2015;271:479-92.

7. Li C, Chen S, Zhou Y, et al. Application of induced pluripotent stem cell transplants: Autologous or allogeneic? Life Sci 2018;212:145-9.

8. Sommer A, Maxreiter F, Krach F, et al. Th17 Lymphocytes induce neuronal cell death in a human iPSC-based model of Parkinson's disease. Cell Stem Cell 2018;23:123-131.e6.

9. Zheng J, Choi KA, Kang PJ, et al. A combination of small molecules directly reprograms mouse fibroblasts into neural stem cells. Biochem Biophys Res Commun 2016;476:42-8.

10. Takahashi A, Nakajima H, Uchida K, et al. Comparison of mesenchymal stromal cells isolated from murine adipose tissue and bone marrow in the treatment of spinal cord injury. Cell Transplant 2018;27:1126-39.

11. Lu P, Wang Y, Graham L, et al. Long-distance growth and connectivity of neural stem cells after severe spinal cordinjury. Cell 2012;150:1264-73.

12. Anderson AJ, Piltti KM, Hooshmand MJ, et al. Preclinical efficacy failure of human CNS-derived stem cells for use in the pathway study of cervical spinal cord injury. Stem Cell Reports 2017;8:249-63.

13. Snyder EY, Teng YD. Stem cells and spinal cord repair. N
Engl J Med 2012;366:1940-2.

14. Libro R, Bramanti P, Mazzon E. The combined strategy of mesenchymal stem cells and tissue-engineered scaffolds for spinal cord injury regeneration. Exp Ther Med 2017;14:3355-68.

15. Yang YW, Wang YL, Lu JK, et al. Delayedxenon postconditioning mitigates spinal cord ischemia/reperfusion injury in rabbits by regulating microglial activation and inflammatory factors. Neural Regen Res 2018;13:510-7.

16. Yun Y, Oh J, Kim Y, et al. Characterization of neural stem cells modified with hypoxia/neuron-specific VEGF expression system for spinal cord injury. Gene Ther 2018;25:27-38.

17. Jin H, Liu ML, Kim HA, et al. Role of the oxygendependent degradation domain in a hypoxia-inducible gene expression system in vascular endothelial growth factor gene therapy. Spine (Phila Pa 1976) 2009;34:E952-8.

18. Assinck P, Duncan GJ, Hilton BJ, et al. Cell transplantation therapy for spinal cord injury. Nat Neurosci 2017;20:637-47.

19. Nutt SE, Chang EA, Suhr ST, et al. Caudalized human iPSC-derived neural progenitor cells produce neurons and glia but fail to restore function in an early chronic spinal cord injury model. Exp Neurol 2013;248:491-503.

20. Oh J, Kim Y, Che L, et al. Regulation of cAMP and GSK3 signaling pathways contributes to the neuronal conversion of glioma. PLoS One 2017;12:e0178881.

21. Azmitia L, Capetian P. Single-step plasmid based reprogramming of human dermal fibroblasts to induced neural stem cells. Methods Mol Biol 2018;1842:31-41.

22. Zhu S, Ambasudhan R, Sun W, et al. Small molecules enable OCT4-mediated direct reprogramming into expandable human neural stem cells. Cell Res 2014;24:126-9.

23. Jain NB, Ayers GD, Peterson EN, et al. Traumatic spinal cord injury in the United States, 1993-2012. JAMA 2015;313:2236-43.

24. Chu T, Zhou H, Lu L, et al. Valproic acid mediated neuroprotection and neurogenesis after spinal cord injury: from mechanism to clinical potential. Regen Med 2015;10:193-209.

25. Rabchevsky AG, Patel SP, Springer JE. Pharmacological interventions for spinal cord injury: where do we stand? How might we step forward? Pharmacol Ther 2011;132:15-29.

26. Oyinbo CA. Secondary injury mechanisms in traumatic spinal cord injury: a nugget of this multiply cascade. Acta 
Neurobiol Exp (Wars) 2011;71:281-99.

27. Niu W, Zang T, Wang LL, et al. Phenotypic reprogramming of striatal neurons into dopaminergic neuron-like cells in the adult mouse brain. Stem Cell Reports 2018;11:1156-70.

28. Park JW, Lee JK, Sheu KM, et al. Reprogramming normal human epithelial tissues to a common, lethal neuro endocrine cancer lineage. Science 2018;362:91-5.

29. Liu ML, Zang T, Zhang CL. Direct lineage reprogramming reveals disease-specific phenotypes of motor neurons from human ALS patients. Cell Rep 2016;14:115-28.

30. Oh J, You Y, Yun Y, et al. A gene and neural stem cell therapy platform based on neuronal cell type-inducible gene overexpression. Yonsei Med J 2015;56:1036-43.

31. Kwon BK, Tetzlaff W, Grauer JN, et al. Pathophysiology and pharmacologic treatment of acute spinal cord injury. Spine J 2004:4:451-64.

32. Twyman RM, Jones EA. Sequences in the proximal 5' flanking region of the rat neuron-specific enolase (NSE) gene are sufficient for cell type-specific reporter gene expression. J Mol Neurosci 1997;8:63-73.

33. Zhang Q, Yan Q, Yang H, et al. Oxygen sensing and adaptability won the 2019 Nobel Prize in Physiology or medicine. Genes Dis 2019;6:328-32.

34. Maxwell PH, Pugh CW, Ratcliffe PJ. Inducible operation of the erythropoietin 3 ' enhancer in multiple cell lines: evidence for a widespread oxygen-sensing mechanism. Proc Natl Acad Sci USA 1993;90:2423-7.

35. Rao MS, Malik N. Assessing iPSC reprogramming methods for their suitability in translational medicine. J Cell Biochem 2012;113:3061-8.

36. Abematsu M, Tsujimura K, Yamano M, et al. Neurons derived from transplanted neural stem cells restore disrupted neuronal circuitry in a mouse model of spinal cord injury. J Clin Invest 2010;120:3255-66.

37. Hu W, Qiu B, Guan W, et al. Direct conversion of normal and Alzheimer's disease human fibroblasts into neuronal cells by small molecules. Cell Stem Cell 2015;17:204-12.

38. Li X, Zuo X, Jing J, et al. Small molecule-driven direct reprogramming of mouse fibroblasts into functional neurons. Cell Stem Cell 2015;17:195-203.

39. Cheng L, Hu W, Qiu B, et al. Generation of neural progenitor cells by chemical cocktails and hypoxia. Cell Res 2014;24:665-79.

40. Zhang L, Yin JC, Yeh H, et al. Small molecules efficiently reprogram human astroglial cells into functional neurons. Cell Stem Cell 2015;17:735-47.
41. Sato N, Meijer L, Skaltsounis L, et al. Maintenance of pluripotency in human and mouse embryonic stem cells through activation of Wnt signalingby a pharmacological GSK-3-specific inhibitor. Nat Med 2004;10:55-63.

42. Yu J, Chau KF, Vodyanik MA, et al. Efficient feeder-free episomal reprogramming with small molecules. PLoS One 2011;6:e17557.

43. Hao Y, Creson T, Zhang L, et al. Mood stabilizer valproate promotes ERK pathway-dependent cortical neuronal growth and neurogenesis. J Neurosci 2004;24:6590-9.

44. Su Z, Niu W, Liu ML, et al. In vivo conversion of astrocytes to neurons in the injured adult spinal cord. Nat Commun 2014;25;5:3338.

45. Kanai H, Sawa A, Chen RW, et al. Valproic acid inhibits histone deacetylase activity and suppresses excitotoxicityinduced GAPDH nuclear accumulation and apoptotic death in neurons. Pharmacogenomics J 2004;4:336-44.

46. Lee JY, Kim HS, Choi HY, et al. Valproic acid attenuates blood-spinal cord barrier disruption by inhibiting matrix metal loprotease-9 activity and improves functional recovery after spinal cord injury. J Neurochem 2012;121:818-29.

47. Kanno H, Ozawa H, Sekiguchi A, et al. The role of autophagy in spinal cord injury. Autophagy 2009;5:390-2.

48. Li W, Sun W, Zhang Y, et al. Rapid induction and longterm self-renewal of primitive neural precursors from human embryonic stem cells by small molecule inhibitors. Proc Natl Acad Sci USA 2011;108:8299-304.

49. Chiu CT, Liu G, Leeds P, et al. Combined treatment with the mood stabilizers lithium and valproate produces multiple beneficial effects in transgenic mouse models of Huntington's disease. Neuropsychopharmacology 2011;36:2406-21.

50. Chiu CT, Wang Z, Hunsberger JG, et al. Therapeutic potential of mood stabilizers lithium and valproic acid: beyond bipolar disorder. Pharmacol Rev 2013;65:105-42.

51. Jin G, Liu B, You Z, et al. Development of a novel neuroprotective strategy: combined treatment with hypothermia andvalproic acid improves survival in hypoxic hippocampal cells. Surgery 2014;156:221-8.

52. Huang HY, Lin SZ, Chen WF, et al. Urocortin modulates dopaminergic neuronal survival via inhibition of glycogen synthase kinase- $3 \beta$ and histone deacetylase. Neurobiol Aging 2011;32:1662-77.

53. Leng $Y$, Liang $M H$, Ren $M$, et al. Synergistic neuroprotective effects of lithium and valproic acid or other histone deacetylase inhibitors in neurons: roles of glycogen synthase kinase-3 inhibition. J Neurosci 
2008;28:2576-88.

54. Setiawan $M$, Tan XW, Goh TW, et al. Inhibiting glycogen synthasekinase- 3 and transforming growth factor- $\beta$ signaling to promote epithelial transition of human adipose mesenchymal stem cells. Biochem Biophys Res Commun

Cite this article as: Pan Z, Oh J, Huang L, Zeng Z, Duan P, Li Z, Yun Y, Kim J, Ha Y, Cao K. The combination of forskolin and VPA increases gene expression efficiency to the hypoxia/ neuron-specific system. Ann Transl Med 2020;8(15):933. doi: 10.21037/atm-20-3871
2017;490:1381-8.

55. Feng B, Ng JH, Heng JC, et al. Molecules that promote or enhance reprogramming of somatic cells to induced pluripotent stem cells. Cell Stem Cell 2009;4:301-12. 
Supplementary

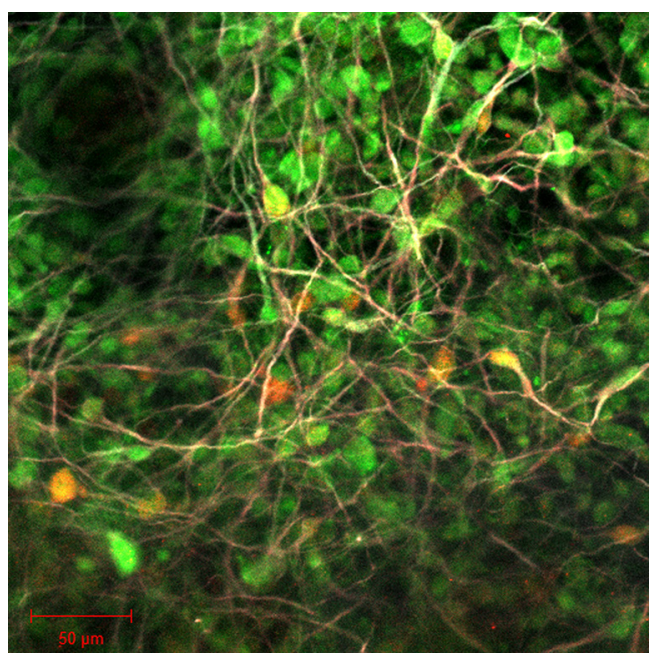

Figure S1 A representative identified image of human induced neural stem cells. 